\title{
Assessment of Antifungal Activities of Phytoextracts against Rhizoctonia solani, Kuhn Causing Sheath Blight of Rice
}

\author{
Swagatika Mohanty ${ }^{1}$, S.S. Mahapatra ${ }^{2}$, A. Khandual ${ }^{3}$, \\ S.K. Sahoo ${ }^{4}$ and A.K. Mukherjee ${ }^{3 *}$ \\ ${ }^{1}$ Department of Plant Pathology, College of Agriculture, Odisha University of Agriculture \& \\ Technology, Bhubaneswar, India \\ ${ }^{2}$ Regional Research \& Transfer of Technology Station (OUAT), Bhadrak, India \\ ${ }^{3}$ Molecular Plant Pathology laboratory, Crop Protection Division, \\ ICAR-NRRI, Cuttack, India \\ ${ }^{4}$ Institute of Life Sciences, Bhubaneswar, India \\ *Corresponding author
}

\section{A B S T R A C T}

\section{Keywords}

Antifungal

activities,

Phytoextracts, Rhizoctonia solani, Sheath blight, Rice

Article Info

Accepted:

05 June 2020

Available Online:

10 July 2020
In vitro evaluation of twelve phytoextracts (aqueous and methanolic extract) of locally available plants having antimicrobial properties were done against Rhizoctonia solani, Kuhn by sequentially highering the concentration of the extracts i.e. at $1 \%, 2.5 \%, 5 \%$ and $10 \%$. The poisoned food technique employed for the purpose.Among all phytoextracts, clove extracts of garlic gave highest inhibition against the fungal growth in all concentrations of its both solvent extracts i.e. $43.23 \%$ to $82.73 \%$ in aqueous extract and $51.50 \%$ to $90.27 \%$ in methanolic extract followed by turmeric and datura respectively. The highest radial growth inhibition was achived by methanolic extract of garlic @ $10 \%$ $(90.27 \%)$ followed by its aqueous extract(82.73\%) and turmeric aqueous extract(65.23\%) respectively in the same concentration. The best performing phytoextracts were tested in vivo in pot culture and in the field(2018 kharif \& 2019 kharif) to confirm their efficacy against the fungus inciting sheath blight in rice. The garlic methanolic extract was treated along with its nano-emulsion in vivo condition and nano-emulsion of methanolic extract was found most effective by reducing the percent disease index in both the years $(2018$ kharif and 2019 kharif). Carbendazim 50WP(11/lit) was used as standard fungicide for comparison.

\section{Introduction}

Rice (Oryza sativa) is the cereal having a world consumption of 450 million tonnes 90\% of this rice consumed in Asia, including the region's 560 million people still affected by hunger (IRRI strategic plan 2017-25). India is the second largest country in terms of production and consumption of rice in the world. In India it is cultivated in 42 million ha (Prasad, 2001). There are about 37 fungal diseases that have been reported in rice $(\mathrm{Ou}$, 
1985). Sheath blight is a fungal disease of rice which economically comes second after blast disease. The fungus belongs to the basidiomycetes group i.e. anastomosis and non-spore forming. It is soil-borne, necrotroph in nature and producing sclerotia in soil for its survival and perpetuation from season to season. This fungus is highly polyphagous in nature and ability to form complexes by intermingling with other anastomosis group that is why no resistant variety sustained against the disease till now. In the present time, the indiscriminate use of synthetic pesticides has created a threat to mankind by polluting the environment and causing other major issues like pest resurgence (minor pest becomes major one), reducing the sustainability of the land in terms of agricultural production. So, moving towards organic farming, use of phytoextracts for plant disease management can be a substitute to the synthetic chemicals. As the botanicals have antimicrobial properties and have the ability to induce resistance in plant against the pathogens, here an attempt has been made to report the botanicals that has been used in these years to manage the particular disease.

\section{Materials and Methods}

Twelve locally available plants having antimicrobial properties were collected, washed properly and air dried. For aqueous extract preparation, $50 \mathrm{~g}$ of plant material along with $50 \mathrm{ml}$ double distilled water $(1: 1 \mathrm{w} / \mathrm{v})$ was taken and grinded. The extract was collected in a muslin cloth and squeezed. The filtrate was collected and centrifuged @ 5000rpm for 10 minutes. The supernatant was collected and passed through coarse filter paper followed by fine filter paper twice. The filtrate was then passed through Tarson's filter apparatus which contains $0.2 \mu \mathrm{m}$ pore size filter paper with the help of a vaccum suction pump to make the final extract bacteria and other microbe free. The concentration of the extract was $100 \%$, the required amount of concentration of the extract was made by diluting the particular extract with required amount of molten potato dextrose agar media. The procedure was practised in sterilized condition. For methanolic extract preparation, the plant materials were subjected to pulverization after shade drying to get coarse powder. The particular plant powder was placed in soxhlet apparatus with methanol $(80 \%)$ and the extraction was done at $55^{\circ} \mathrm{C}$ for 6 hours. Then, the extract was placed in rotary evaporator @ $120 \mathrm{rpm}$ at $227-228 \mathrm{mp}$ vaccum in $45^{\circ} \mathrm{C}$ for 5 hours. The final crude extract obtained after the extraction was taken as stock solution $(100 \%)$ and further dilution was made by adding the required amount molten media. For preparation of nano-methanolic emulsion, $1 \mathrm{ml}$ of garlic methanolic extract was mixed with $1 \mathrm{ml}$ of Tween 20 and $8 \mathrm{ml}$ of distilled water by vortexing ( 2 minutes).

After vortexing, the tube containing the mixture was placed inside sonicator $(37 \%$ ampl., room temperature) for 10 minutes for formation of nano-emulsion. For stability checking, phase separation and particle size analyzer was used.

Preliminary screening of the phytoextracts were made by Poisoned food technique (Vincent, 1947). 90mm petriplates containing $20 \mathrm{ml}$ of poisoned potato dextrose agar (PDA) media were used. Calculated amount of extracts were mixed with the molten media and plating was done for solidification. For each treatment, three replications were maintained. $5 \mathrm{~mm}$ disc of hyphal mat was cut from edges of 5 days old culture plate of fungus and inoculated at the centre of the poisoned plate and incubated at $27 \pm 1^{\circ} \mathrm{C}$. The radial growth measurement of the fungus was done in regular interval till the control plate showed the full growth. The efficacy of the 
phyto extracts was expressed by per cent radial growth inhibition over the control which was determined by the formula(Vincent,1947)

$\%$ Radial growth inhibition $(\mathrm{I})=$

Radial growth in control (C) - Radial growth in treatment $(\mathrm{T}) \mathrm{X} 100$

Radial growth in control (C)

The best performing phytoextracts of in vitro study were tested in pot culture and in field condition to know their efficacy in seminatural and natural environment. In pot culture experiment, $(45 \mathrm{~cm}$ X $30 \mathrm{~cm})$ sized pot was used. Each pot contained $4 \mathrm{~kg}$ of soil. 21 days old seedling was transplanted in each pot. Three number of hills were maintained in each pot. After 25 days of transplanting, three primary tillers were selected and inoculated with $5 \mathrm{~mm}$ mycelial disc cut from edges of 7 days old fresh culture plate. In order to avoid the falling of disc from sheath, the disc was wrapped with wet cotton around the sheath. Two sets of treatments were maintained. In one set, there were pre-inoculation spray of treatments before 24 hours of inoculation and in another; there were post-inoculation spray after 24 hours of the inoculation. The spraying was done by atomizer. Since, the pathogen requires high humidity for infection initiation, spraying of water was done every day near the point of inoculation of the plants. In control pots, solvents were sprayed to evaluate whether the solvent has any hazardous impact on plant or not. Absolute control was maintained and spray with $1 \%$ carbendazim was done for comparative study. Data was recorded in each 7 days interval after first appearance of the disease symptom i.e. after 3 days of inoculation(DAI) upto 17 DAI. Here, Relative Vertical Spread (RVS) was taken in to account for disease severity study. The vertical spread and total plant height were measured and relative vertical spread (RVS) was calculated using the formula given by Ahn et al., (1986).

$$
\text { RVS }=\frac{\text { Vertical spread }}{\text { Plant height }} \times 100
$$

Rice sheath blight grade chart (IRRI, 1996)

Disease Vertical spread grade

$0 \quad$ No infection observed

1 Lesion limited to lower 20 per cent of the height of the plant

3 Lesion limited to 21-30 per cent of the height of the plant

5 Lesion limited to 31-45 per cent of the height of the plant

7 Lesion limited to 46-65 per cent of the height of the plant

9 Lesion more than 65 per cent of the height of the plant

Field experiment was conducted in Randomized Block Design. Each treatment had three replication. ( $6 \mathrm{~m}$ X $3 \mathrm{~m})$ sized plot were taken for each replication. Judicious use of fertilizer was used and water level was maintained up to optimum level to facilitate disease initiation. In field, inoculation was done in 'straw bead method' i.e., at the active tillering phase of the plants. Like pot culture experiment, two sets of treatments were maintained for pre-inoculation spray and postinoculation spray. Sprays were done as per pot culture experiment. Data was recorded before the spray and in each 10 days interval after inoculation up to 30 days. For disease severity scoring was recorded by following the scale of SES, IRRI, 1996. Per cent Disease Index (Wheeler, 1969) and Grain Yield (kg/ha) were the parameters which taken in to consideration for data analysis.

Per cent Disease Index $=$

Sum of rating $\mathrm{X}$ 100 Number of plants observed Maximum Scale 


\section{Results and Discussion}

Primary screening of the phytoextracts with two different solvents(aqueous and methanol) was done by poisoned food technique. Initially $12 \mathrm{X} 2=22$ plant extracts were tested in four concentration i.e. $1 \%, 2.5 \%, 5 \%$ and $10 \%$. Among all extracts methanolic extract of garlic@10\% was shown the highest inhibition of radial growth i.e. $84.67 \%$ followed by garlic aqueous extract (82.73\%), turmeric aqueous extract $(65.23 \%)$ and datura (Datura metel) aqueous extract (53.10\%) respectively at that same concentration. In vitro study revealed that both the extracts of garlic in all concentrations showed significant superiority than other phyto extracts after 72 hours of inoculation (Table 1). The garlic (Allium sativum) extract also restricted the formation of sclerotia in the plates. This finding is in agreement with the work of Kumar et al., (2017) who found garlic bulb extract gave highest radial growth inhibition of Rhizoctinia solani (80.19\%) @ 10\% and also with the finding of Yadav (2007). The nano-emulsion of methanlolic extract and methanolic extract of garlic has the significant effect on inhibition of the fungus. This implies methanol is a better solvent for consistant extraction of active ingredients of the plant extracts as compared to other solvent as reported by Lin et al., (1999) and Eloff et al., (1998). In pot experiment and field experiment, pre-inoculation spray of phytoextracts was given better result than the post-inoculation spray(Table 2 and Table 3 ). This result corroborates with the finding of Choudhary et al., (2017) who also found the pre-inoculation spray of botanicals, specially clerodendrum choloform extract had given highest inhibition of disease in green house as well as in field condition. The nano-emulsion of methanolic extract of garlic @ 1\% $(10,000 \mathrm{ppm})$ was given significant reduction in percent disease index $31.15 \%$ over control was suggested that nano-emusion is more target specific and consumes less active ingredient of the phytoextract for effective result. This finding is in confirmation with the works of Ali et al., (2017) who found the nano-emulsion of neem and citronella oils most effective against Rhizoctonia solani and Sclerotium rolfsii in vitro. After garlic extract, turmeric (Curcuma longa) aqueous extract $(10 \%)$ was the second effective extract against the fungus in pot culture (RVS ranges from $24.04 \%$ to $32.79 \%$ ).

Methanol extracts of several plants of Zingiberaceae family showed great potential as antifungal and antibacterial properties (Yusuf et al., 2001), little deviates from the present findings as the extracts of turmeric also belonged to the same family but the aqueous extract works better. In the field condition the effect of turmeric and datura aqueous extract were at par i.e. $16.49 \%$ and $18.50 \%$ over control respectively. The effect of datura(Datura metel) was not significant on disease control. This finding is in contrast to the reports of Kagale et al., (2004), Bhattacharya et al., (2013). Kagale et al., (2004) found methanolic extract of Datura metel inducing systemic resistance against $R$.solani and Bhattacharya et al., also reported that ethanolic extract of Datura metel induced systemic resistance in rice against sheath blight fungus.

From the experiment, it was found that extracts of garlic(Allium sativum) was the most effective in managing the sheath blight fungus in a greater extent. It may be because of the biologically active compounds present in it which affect a wide range of soil-borne fungal pathogens as reported by Kyung and Lee(2001). Nano emulsion of garlic methanolic extract has the potential to manage the disease in a greates extent as it is target specific and ecofriendly and biodegradable. 
Table.1 In vitro evaluation of plant extracts against Rhizoctonia solani

\begin{tabular}{|c|c|c|c|c|c|c|c|c|c|c|}
\hline \multirow{3}{*}{$\begin{array}{l}\text { Sl. } \\
\text { No }\end{array}$} & \multirow{3}{*}{ Plant Extract } & \multirow{3}{*}{ Plant Parts used } & \multicolumn{8}{|c|}{ Per cent Radial growth Inhibition(\%) of Rhizoctonia solani } \\
\hline & & & \multicolumn{2}{|c|}{$1 \%$} & \multicolumn{2}{|c|}{$2.5 \%$} & \multicolumn{2}{|c|}{$5 \%$} & \multicolumn{2}{|c|}{$10 \%$} \\
\hline & & & $\mathbf{A}$ & M & $\mathbf{A}$ & M & $\mathbf{A}$ & M & $\mathbf{A}$ & $\mathbf{M}$ \\
\hline 1 & $\begin{array}{l}\text { Ginger } \\
\text { (Zingiber officinalae) }\end{array}$ & Rhizome & $\begin{array}{c}17.27 \\
(24.55)\end{array}$ & $\begin{array}{l}10.50 \\
(18.9)\end{array}$ & $\begin{array}{c}28.30 \\
(32.13)\end{array}$ & $\begin{array}{c}17.63 \\
(24.83)\end{array}$ & $\begin{array}{c}35.30 \\
(36.44)\end{array}$ & $\begin{array}{c}23.80 \\
(29.18)\end{array}$ & $\begin{array}{c}40.57 \\
(39.56)\end{array}$ & $\begin{array}{c}31.90 \\
(34.38)\end{array}$ \\
\hline 2 & $\begin{array}{l}\text { Kalmegh } \\
\text { (Andrographis paniculata) }\end{array}$ & Leaves & $\begin{array}{c}6.80 \\
(15.11)\end{array}$ & $\begin{array}{c}5.60 \\
(13.67)\end{array}$ & $\begin{array}{c}9.27 \\
(17.72)\end{array}$ & $\begin{array}{c}7.57 \\
(15.95)\end{array}$ & $\begin{array}{l}11.97 \\
(20.21)\end{array}$ & $\begin{array}{c}9.30 \\
(17.71)\end{array}$ & $\begin{array}{c}17.53 \\
(24.75)\end{array}$ & $\begin{array}{c}13.23 \\
(21.33)\end{array}$ \\
\hline 3 & $\begin{array}{l}\text { Clerodendrum } \\
\text { (Clerodendrum } \\
\text { infortunatum) }\end{array}$ & Leaves & $\begin{array}{c}5.30 \\
(13.31)\end{array}$ & $\begin{array}{c}8.70 \\
(17.15)\end{array}$ & $\begin{array}{c}11.20 \\
(19.49)\end{array}$ & $\begin{array}{c}13.70 \\
(21.66)\end{array}$ & $\begin{array}{c}13.87 \\
(21.86)\end{array}$ & $\begin{array}{c}17.80 \\
(24.95)\end{array}$ & $\begin{array}{c}17.27 \\
(24.55)\end{array}$ & $\begin{array}{c}25.77 \\
(30.50)\end{array}$ \\
\hline 4 & $\begin{array}{l}\text { Carrot grass } \\
\text { (Parthenium } \\
\text { hysterophorus) }\end{array}$ & Leaves & $\begin{array}{c}13.40 \\
(21.44)\end{array}$ & $\begin{array}{c}2.80 \\
(9.63)\end{array}$ & $\begin{array}{l}24.60 \\
(29.76)\end{array}$ & $\begin{array}{c}3.90 \\
(11.29)\end{array}$ & $\begin{array}{c}27.27 \\
(31.43)\end{array}$ & $\begin{array}{c}7.27 \\
(15.63)\end{array}$ & $\begin{array}{l}28.13 \\
(32.01)\end{array}$ & $\begin{array}{l}13.67 \\
(21.7)\end{array}$ \\
\hline 5 & $\begin{array}{l}\text { Neem } \\
\text { (Azadirachta indica) }\end{array}$ & Leaves & $\begin{array}{c}2.70 \\
(9.46)\end{array}$ & $\begin{array}{c}7.87 \\
(16.28)\end{array}$ & $\begin{array}{c}7.67 \\
(16.42)\end{array}$ & $\begin{array}{c}11.93 \\
(16.32)\end{array}$ & $\begin{array}{c}9.13 \\
(17.89)\end{array}$ & $\begin{array}{c}19.53 \\
(26.22)\end{array}$ & $\begin{array}{c}11.60 \\
(19.90)\end{array}$ & $\begin{array}{c}25.83 \\
(24.78)\end{array}$ \\
\hline 6 & $\begin{array}{l}\text { Tulsi } \\
\text { (Ocimum sanctum) }\end{array}$ & Leaves & $\begin{array}{c}10.27 \\
(18.69)\end{array}$ & $\begin{array}{c}2.87 \\
(9.72)\end{array}$ & $\begin{array}{c}17.80 \\
(24.95)\end{array}$ & $\begin{array}{c}9.57 \\
(17.97)\end{array}$ & $\begin{array}{c}20.40 \\
(26.83)\end{array}$ & $\begin{array}{c}14.80 \\
(22.58)\end{array}$ & $\begin{array}{c}23.20 \\
(28.78)\end{array}$ & $\begin{array}{c}17.57 \\
(34.96)\end{array}$ \\
\hline 7 & $\begin{array}{l}\text { Begunia } \\
\text { (Vitex negundo) }\end{array}$ & Leaves & $\begin{array}{c}9.23 \\
(17.67)\end{array}$ & $\begin{array}{c}11.53 \\
(19.85)\end{array}$ & $\begin{array}{c}13.80 \\
(21.80)\end{array}$ & $\begin{array}{c}17.43 \\
(24.62)\end{array}$ & $\begin{array}{c}22.23 \\
(28.11)\end{array}$ & $\begin{array}{c}28.73 \\
(32.41)\end{array}$ & $\begin{array}{c}24.20 \\
(29.46)\end{array}$ & $\begin{array}{c}32.83 \\
(72.14)\end{array}$ \\
\hline 8 & $\begin{array}{l}\text { Garlic } \\
\text { (Allium sativum) }\end{array}$ & Cloves & $\begin{array}{c}43.23 \\
(41.11)\end{array}$ & $\begin{array}{c}51.50 \\
(45.86)\end{array}$ & $\begin{array}{c}61.60 \\
(51.71)\end{array}$ & $\begin{array}{l}72.83 \\
(58.6)\end{array}$ & $\begin{array}{c}76.57 \\
(61.12)\end{array}$ & $\begin{array}{c}84.67 \\
(72.79)\end{array}$ & $\begin{array}{c}82.73 \\
(65.48)\end{array}$ & $\begin{array}{c}90.27 \\
(72.14)\end{array}$ \\
\hline 9 & $\begin{array}{l}\text { Turmeric } \\
\text { (Curcuma longa) }\end{array}$ & Rhizome & $\begin{array}{c}16.20 \\
(23.73)\end{array}$ & $\begin{array}{c}11.17 \\
(19.45)\end{array}$ & $\begin{array}{c}34.23 \\
(35.81)\end{array}$ & $\begin{array}{c}20.43 \\
(26.87)\end{array}$ & $\begin{array}{c}43.50 \\
(41.25)\end{array}$ & $\begin{array}{c}37.47 \\
(37.72)\end{array}$ & $\begin{array}{c}65.23 \\
(53.88)\end{array}$ & $\begin{array}{c}50.10 \\
(44.02)\end{array}$ \\
\hline 10 & $\begin{array}{l}\text { Datura } \\
\text { (Datura metel) }\end{array}$ & Leaves & $\begin{array}{c}13.73 \\
(21.74)\end{array}$ & $\begin{array}{c}9.37 \\
(17.80)\end{array}$ & $\begin{array}{c}22.17 \\
(28.08)\end{array}$ & $\begin{array}{c}15.27 \\
(22.92)\end{array}$ & $\begin{array}{c}32.60 \\
(34.81)\end{array}$ & $\begin{array}{c}19.27 \\
(26.02)\end{array}$ & $\begin{array}{c}53.10 \\
(46.78)\end{array}$ & $\begin{array}{c}31.47 \\
(34.09)\end{array}$ \\
\hline 11 & $\begin{array}{l}\text { Ber/Common jujube } \\
\text { (Zizyphus jujuba) }\end{array}$ & Leaves & $\begin{array}{c}5.80 \\
(13.93)\end{array}$ & $\begin{array}{c}2.97 \\
(9.88)\end{array}$ & $\begin{array}{c}9.20 \\
(17.66)\end{array}$ & $\begin{array}{c}5.73 \\
(13.74)\end{array}$ & $\begin{array}{c}11.63 \\
(19.94)\end{array}$ & $\begin{array}{c}9.97 \\
(18.37)\end{array}$ & $\begin{array}{c}14.40 \\
(22.27)\end{array}$ & $\begin{array}{c}13.57 \\
(21.61)\end{array}$ \\
\hline 12 & $\begin{array}{l}\text { Pink morning glory } \\
\text { (Ipomoea carnea) }\end{array}$ & Leaves & $\begin{array}{c}7.47 \\
(15.85)\end{array}$ & $\begin{array}{c}9.13 \\
(17.57)\end{array}$ & $\begin{array}{c}12.73 \\
(20.89)\end{array}$ & $\begin{array}{c}14.63 \\
(22.45)\end{array}$ & $\begin{array}{c}15.43 \\
(23.13)\end{array}$ & $\begin{array}{c}17.83 \\
(24.97)\end{array}$ & $\begin{array}{c}17.43 \\
(24.68)\end{array}$ & $\begin{array}{c}21.87 \\
(27.87)\end{array}$ \\
\hline 13 & Control & - & 0.00 & 0.00 & 0.00 & 0.00 & 0.00 & 0.00 & 0.00 & 0.00 \\
\hline \multirow{2}{*}{\multicolumn{3}{|c|}{$\begin{array}{l}\text { SEm } \\
\text { CD at } 5 \%\end{array}$}} & 0.29 & 0.60 & 0.43 & 1.40 & 0.95 & 1.50 & 0.72 & 0.96 \\
\hline & & & 0.86 & 1.76 & 1.25 & 4.08 & 2.77 & 4.38 & 2.12 & 2.81 \\
\hline
\end{tabular}


Table.2 Pot culture experiment of plant extracts against Rhizoctonia solani

\begin{tabular}{|c|c|c|c|c|c|c|c|}
\hline \multirow[b]{2}{*}{$\begin{array}{l}\text { Sl. } \\
\text { No. }\end{array}$} & \multirow{2}{*}{$\begin{array}{c}\text { Treatment details } \\
\text { Pre-inoculation Spray }\end{array}$} & \multicolumn{3}{|c|}{ Relative Vertical Spread(\%) } & \multicolumn{3}{|c|}{$\begin{array}{l}\text { Per cent reduction over } \\
\text { control }(\%)\end{array}$} \\
\hline & & $\begin{array}{c}3 \\
\text { DAI }\end{array}$ & $\begin{array}{c}10 \\
\text { DAI }\end{array}$ & $\begin{array}{c}17 \\
\text { DAI }\end{array}$ & $\begin{array}{c}3 \\
\text { DAI }\end{array}$ & $\begin{array}{c}10 \\
\text { DAI }\end{array}$ & $\begin{array}{l}17 \\
\text { DAI }\end{array}$ \\
\hline 1 & Spray with garlic aqueous extract@ 10\% & $\begin{array}{c}10.11 \\
(18.54)\end{array}$ & $\begin{array}{c}21.4 \\
(27.56)\end{array}$ & $\begin{array}{c}26.31 \\
(30.86)\end{array}$ & $\begin{array}{c}43.26 \\
(41.13)\end{array}$ & $\begin{array}{c}40.06 \\
(39.26)\end{array}$ & $\begin{array}{c}37.24 \\
(37.60)\end{array}$ \\
\hline 2 & Spray with garlic methanolic extract@10\% & $\begin{array}{c}8.80 \\
(17.26)\end{array}$ & $\begin{array}{c}19.42 \\
(26.15)\end{array}$ & $\begin{array}{c}23.90 \\
(29.27)\end{array}$ & $\begin{array}{c}48.82 \\
(44.38)\end{array}$ & $\begin{array}{c}44.41 \\
(41.79)\end{array}$ & $\begin{array}{c}42.96 \\
(40.96)\end{array}$ \\
\hline 3 & Spray with garlic methanolic nano-emulsion@1\% & $\begin{array}{c}7.10 \\
(15.45)\end{array}$ & $\begin{array}{c}15.71 \\
(23.35)\end{array}$ & $\begin{array}{c}19.50 \\
(26.21)\end{array}$ & $\begin{array}{c}58.24 \\
(49.74)\end{array}$ & $\begin{array}{c}55.01 \\
(47.88)\end{array}$ & $\begin{array}{c}53.46 \\
(46.99)\end{array}$ \\
\hline 4 & Spray with turmeric aqueous extract @ 10\% & $\begin{array}{c}10.50 \\
(18.91)\end{array}$ & $\begin{array}{c}23.40 \\
(28.93)\end{array}$ & $\begin{array}{c}28.16 \\
(32.05)\end{array}$ & $\begin{array}{c}41.01 \\
(39.83)\end{array}$ & $\begin{array}{c}34.45 \\
(35.94)\end{array}$ & $\begin{array}{c}32.79 \\
(34.93)\end{array}$ \\
\hline 5 & Spray with ginger aqueous extract @ 10\% & $\begin{array}{c}12.30 \\
(20.53)\end{array}$ & $\begin{array}{c}28.30 \\
(32.14)\end{array}$ & $\begin{array}{c}37.80 \\
(37.94)\end{array}$ & $\begin{array}{c}30.90 \\
(33.77)\end{array}$ & $\begin{array}{c}20.73 \\
(27.08)\end{array}$ & $\begin{array}{c}11.48 \\
(19.80)\end{array}$ \\
\hline 6 & Spray with datura aqueous extract @10\% & $\begin{array}{c}11.30 \\
(19.64)\end{array}$ & $\begin{array}{c}24.80 \\
(29.87)\end{array}$ & $\begin{array}{c}31.80 \\
(34.33)\end{array}$ & $\begin{array}{c}36.52 \\
(37.29)\end{array}$ & $\begin{array}{c}30.53 \\
(33.70)\end{array}$ & $\begin{array}{c}25.53 \\
(30.35)\end{array}$ \\
\hline 7 & Spray with water(C) & $\begin{array}{c}17.8 \\
(24.95)\end{array}$ & $\begin{array}{c}35.70 \\
(36.68)\end{array}$ & $\begin{array}{c}42.7 \\
(40.80)\end{array}$ & 0 & 0 & 0 \\
\hline 8 & Spray with solvent(C) & $\begin{array}{c}17.2 \\
(24.50)\end{array}$ & $\begin{array}{c}34.93 \\
(36.22)\end{array}$ & $\begin{array}{c}41.9 \\
(40.37)\end{array}$ & 0 & 0 & 0 \\
\hline 9 & Absolute Control(No inoculation, No spray) & 0 & 0 & 0 & 0 & 0 & 0 \\
\hline 10 & Spray with Carbendazim 50WP(1g/lit.) & 0 & 0 & 0 & 0 & 0 & 0 \\
\hline \multicolumn{2}{|c|}{ SEm } & 0.38 & 0.90 & 1.17 & & & \\
\hline \multicolumn{2}{|c|}{ CD at $5 \%$} & 1.13 & 2.67 & 3.45 & & & \\
\hline
\end{tabular}


Table.3 Pot culture experiment of plant extracts against Rhizoctonia solani

\begin{tabular}{|c|c|c|c|c|c|c|c|}
\hline \multirow[t]{2}{*}{$\begin{array}{l}\text { Sl. } \\
\text { No. }\end{array}$} & \multirow{2}{*}{$\begin{array}{c}\text { Treatment details } \\
\text { Post-inoculation Spray }\end{array}$} & \multicolumn{3}{|c|}{$\begin{array}{l}\text { Relative Vertical } \\
\text { Spread }(\%)\end{array}$} & \multicolumn{3}{|c|}{$\begin{array}{l}\text { Per cent inhibition over } \\
\text { control }(\%)\end{array}$} \\
\hline & & $\begin{array}{c}3 \\
\text { DAI }\end{array}$ & $\begin{array}{c}10 \\
\text { DAI }\end{array}$ & $\begin{array}{c}17 \\
\text { DAI }\end{array}$ & $\begin{array}{c}3 \\
\text { DAI }\end{array}$ & $\begin{array}{c}10 \\
\text { DAI }\end{array}$ & $\begin{array}{c}17 \\
\text { DAI }\end{array}$ \\
\hline 1 & Spray with garlic aqueous extract@ 10\% & $\begin{array}{c}10.47 \\
(18.86)\end{array}$ & $\begin{array}{c}22.10 \\
(28.04)\end{array}$ & $\begin{array}{c}30.27 \\
(33.36)\end{array}$ & $\begin{array}{c}41.42 \\
(40.06)\end{array}$ & $\begin{array}{c}38.10 \\
(38.12)\end{array}$ & $\begin{array}{c}29.12 \\
(32.66)\end{array}$ \\
\hline 2 & Spray with garlic methanolic extract@ 10\% & $\begin{array}{c}9.23 \\
(17.62)\end{array}$ & $\begin{array}{c}22.40 \\
(28.23)\end{array}$ & $\begin{array}{c}29.10 \\
(32.64)\end{array}$ & $\begin{array}{c}46.42 \\
(42.95)\end{array}$ & $\begin{array}{c}15.15 \\
(22.88)\end{array}$ & $\begin{array}{c}30.44 \\
(33.47)\end{array}$ \\
\hline 3 & Spray with garlic methanolic nano-emulsion@ 1\% & $\begin{array}{c}8.27 \\
(16.70)\end{array}$ & $\begin{array}{c}14.20 \\
(22.12)\end{array}$ & $\begin{array}{c}23.80 \\
(29.18)\end{array}$ & $\begin{array}{c}52.03 \\
(46.16)\end{array}$ & $\begin{array}{c}46.21 \\
(42.83)\end{array}$ & $\begin{array}{c}43.11 \\
(41.04)\end{array}$ \\
\hline 4 & Spray with turmeric aqueous extract @ 10\% & $\begin{array}{c}14.23 \\
(22.10)\end{array}$ & $\begin{array}{c}25.30 \\
(30.22)\end{array}$ & $\begin{array}{c}32.43 \\
(34.69)\end{array}$ & $\begin{array}{c}20.34 \\
(26.79)\end{array}$ & $\begin{array}{c}29.13 \\
(32.67)\end{array}$ & $\begin{array}{c}24.04 \\
(29.36)\end{array}$ \\
\hline 5 & Spray with ginger aqueous extract @ 10\% & $\begin{array}{c}13.90 \\
(21.89)\end{array}$ & $\begin{array}{c}31.20 \\
(33.95)\end{array}$ & $\begin{array}{c}40.10 \\
(39.29)\end{array}$ & $\begin{array}{c}22.20 \\
(28.11)\end{array}$ & $\begin{array}{c}12.61 \\
(20.80)\end{array}$ & $\begin{array}{c}6.09 \\
(14.19)\end{array}$ \\
\hline 6 & Spray with datura aqueous extract @ 10\% & $\begin{array}{c}14.77 \\
(22.59)\end{array}$ & $\begin{array}{c}29.50 \\
(32.89)\end{array}$ & $\begin{array}{c}35.83 \\
(36.70)\end{array}$ & $\begin{array}{c}17.35 \\
(24.62)\end{array}$ & $\begin{array}{c}17.37 \\
(24.63)\end{array}$ & $\begin{array}{c}16.08 \\
(23.64)\end{array}$ \\
\hline 7 & Spray with water(C) & $\begin{array}{c}17.87 \\
(24.97)\end{array}$ & $\begin{array}{c}35.70 \\
(36.71)\end{array}$ & $\begin{array}{c}42.70 \\
(40.79)\end{array}$ & 0 & 0 & 0 \\
\hline 8 & Spray with solvent(C) & $\begin{array}{c}17.23 \\
(24.53)\end{array}$ & $\begin{array}{c}26.40 \\
(30.87)\end{array}$ & $\begin{array}{c}41.83 \\
(40.29)\end{array}$ & 0 & 0 & 0 \\
\hline 9 & Absolute Control(No inoculation, No spray) & 0 & 0 & 0 & 0 & 0 & 0 \\
\hline 10 & Spray with Carbendazim 50WP(1g/lit.) & 0 & 0 & 0 & 0 & 0 & 0 \\
\hline \multicolumn{2}{|c|}{ SEm } & 0.73 & 0.90 & 1.20 & & & \\
\hline \multicolumn{2}{|c|}{ CD at $5 \%$} & 2.15 & 2.65 & 3.55 & & & \\
\hline
\end{tabular}


Table.4 Field experiment on efficacy of plant extracts against Rhizoctonia solani

\begin{tabular}{|c|c|c|c|c|c|c|c|c|c|}
\hline \multirow[t]{2}{*}{$\begin{array}{l}\text { Sl. } \\
\text { No }\end{array}$} & \multirow[t]{2}{*}{ Plant Extracts } & \multicolumn{3}{|c|}{ Per cent Disease Index(PDI) } & \multirow[t]{2}{*}{$\begin{array}{l}\% \text { reduction } \\
\text { over Control }\end{array}$} & \multicolumn{3}{|c|}{ Yield (kg/ha) } & \multirow{2}{*}{$\begin{array}{c}\% \text { Yield } \\
\text { increase over } \\
\text { control }\end{array}$} \\
\hline & & $\begin{array}{c}2018 \\
\text { kharif }\end{array}$ & $\begin{array}{l}2019 \\
\text { kharif }\end{array}$ & Pooled & & $\begin{array}{c}2018 \\
\text { kharif }\end{array}$ & $\begin{array}{c}2019 \\
\text { kharif }\end{array}$ & Pooled & \\
\hline 1 & Spray with garlic aqueous extract & $\begin{array}{r}35.13 \\
(36.63)\end{array}$ & $\begin{array}{r}36.33 \\
(37.07)\end{array}$ & 36.70 & 18.50 & 1774.67 & 1777.67 & 1776.17 & 13.18 \\
\hline 2 & Spray with garlic methanolic extract & $\begin{array}{r}32.77 \\
(36.57)\end{array}$ & $\begin{array}{l}33.27 \\
(35.22)\end{array}$ & 35.05 & 21.90 & 2050.67 & 1975.33 & 2013.00 & 24.87 \\
\hline 3 & $\begin{array}{l}\text { Spray with garlic methanolic nano- } \\
\text { emulsion }\end{array}$ & $\begin{array}{r}25.43 \\
(31.88)\end{array}$ & $\begin{array}{r}27.40 \\
(31.55)\end{array}$ & 30.90 & 31.15 & 2246.00 & 2440.00 & 2343.00 & 31.20 \\
\hline 4 & Spray with turmeric aqueous extract & $\begin{array}{r}38.50 \\
(33.83)\end{array}$ & $\begin{array}{r}38.70 \\
(38.47)\end{array}$ & 37.48 & 16.49 & 1678.33 & 2175.67 & 1927.00 & 24.97 \\
\hline 5 & Spray with datura aqueous extract & $\begin{array}{r}40.90 \\
(40.34)\end{array}$ & $\begin{array}{r}43.80 \\
(41.44)\end{array}$ & 40.59 & 9.86 & 1577.33 & 1744.33 & 1660.83 & 7.6 \\
\hline 6 & Spray with Carbendazim & $\begin{array}{r}18.60 \\
(26.42)\end{array}$ & $\begin{array}{r}18.90 \\
(25.77)\end{array}$ & 25.63 & & 2631.33 & 2754.33 & 2692.83 & 13.18 \\
\hline 7 & Spray with water(C) & $\begin{array}{r}49.73 \\
(47.01)\end{array}$ & $\begin{array}{r}50.40 \\
(45.23)\end{array}$ & 45.03 & & 1553.00 & 1531.67 & 1542.33 & \\
\hline 8 & Spray with solvent(C) & $\begin{array}{r}49.00 \\
(43.68)\end{array}$ & $\begin{array}{l}51.20 \\
(45.69)\end{array}$ & 44.88 & & 1495.00 & 7320.33 & 1612.00 & \\
\hline \multicolumn{2}{|c|}{ SEm } & 3.84 & 1.10 & 0.85 & & 57.65 & 55.53 & 62.98 & \\
\hline \multicolumn{2}{|c|}{$\mathrm{CD}(5 \%)$} & 11.63 & 3.32 & 2.47 & & 171.26 & 164.97 & 182.59 & \\
\hline
\end{tabular}


There is need of further study of active ingredients present in phyto extracts responsible for inhibiting the growth of the fungus and attempts should to made to know and enhance the shelf life and stability of the extracts so that commercial production of the ecofriendly products can be possible for future use of the farmers for sustainable agriculture.

\section{References}

Ahn, S.W., DenaDela, R.C., Candole, B.L. and Mew, T.W. (1986). A new scale for rice sheath blight disease assessment. Int . Rice Res. Newsl. 11: 1

Ali OMA, Shakil NA, Rana VS, Sarkar DJ, Majumdar S, Kaushik P, Singh BB and Kumar J.2017. Antifungal activity of nano emulsions of neem and citronella oils against phytopathogenic fungi, Rhizoctonia solani and Sclerotium rolfsii

Bhattacharya S, Chahraborty K and Pal TK.2013. Induction of systemic resistance in rice by leaf extracts of Datura metel against sheath blight disease, Archives of Phytopathology and Plant Protection, DOI:10.1080/03235408.2013.792537

Choudhary D, Anand YR, Kundu S, Nath R, Kole RK, Saha J.2017. Effect of plant extracts against sheath blight of rice caused by Rhizoctonia solani, Journal of Pharmacognosy and Phytochemistry, 6(4):399-404

IRRI (1996). Standard evaluation system for the INGER Genetic Resource center, 4th edn.

Kagale S, Marimuthu T, Thayumanavan B, Nandakumar R, Samiyappan R.2004. Antimicrobial activity and induction of systemic resistance in rice by leaf extract of Datura metel against Rhizoctonia solani and Xanthomonas oryzae pv. oryzae,
Physiological and Molecular Plant Pathology,65(2):91-100.

Karthik SR, Sajeena A, Girija JJ, Heera G.2017. Antifungal activities of organic preparations, botanicals and non-hazardous chemicals against Rhizoctonia solani Kuhn causing sheath blight of rice, Journal of Tropical Agriculture, 55(1):104-113

Kumar V, Chaudhary VP, Kumar D, Kumar A,Sagar S, Chaudhary S.2017.Efficacy of botanicals and fungicides against Rhizoctonia solani inciting sheath blight disease on rice(Oryza sativa), Journal of Applied and Natural science, 9(4):1916-1920

Ou SH.1985. Rice diseases, 2nd Edition. Common Wealth Mycological Institute, Kew, Surrey, England. 379

Prasad GSV, Prasadrao U, Shobha Rani N, Rao LVS, Pasalu IC, Muralidharan K.2001.Indian varietes released in countries around the world. Current Science, 8(12):1508-1511.

Vincent,J.M.(1947). Distortion of fungal hyphae in presence of certain inhibitions. Nature,159850

Yusuf NA, Ibrahin H, Khalid N. Antibacterial evaluation and tissue culture studies of selected medicinal cucurma species. (Workshop transcripts) 2001, N.S.F.

Lin J, Opoku AR, Geheeb-Keller M, Hutchings $\mathrm{AD}$, Terblanche SE, Jager $\mathrm{AK}$, et al., Preliminary screening of some traditional Zulu medicinal plants for anti- inflammatory and anti-microbial activities. J. Ethnopharmacol. 1999; 68:267-274.

Eloff JN. Which extract should be used for the screening and isolation of antimicrobial components from plants? J. Ethnopharmacol. 1998; 60:1-8

Yadav, B.C. Gupta, R. P. and Singh, R.V. 2007. Compara- tive performance of Trichoderma spp. as seed dresser and soil application against Fusarium wilt of Pigeonpea. J. Mycol. Pl. Pathol. 35 (3): 541.

\section{How to cite this article:}

Swagatika Mohanty, S.S. Mahapatra, A. Khandual, S.K. Sahoo and Mukherjee, A.K.. 2020. Assessment of Antifungal Activities of Phytoextracts against Rhizoctonia solani, Kuhn Causing Sheath Blight of Rice. Int.J.Curr.Microbiol.App.Sci. 9(07): 01-09.

doi: https://doi.org/10.20546/ijcmas.2020.907.001 